\title{
Integrando ferramentas de auxílio ao diagnóstico no sistema de arquivamento e comunicação de imagens*
}

\author{
Integrating computer-aided diagnosis tools into the picture archiving and communication system
}

Samuel Covas Salomão ${ }^{1}$, Paulo Mazzoncini de Azevedo Marques ${ }^{2}$

Resumo Objetivo: Este artigo apresenta um modelo de integração de algoritmos de diagnóstico auxiliado por computador dentro do fluxo de trabalho dos sistemas de gerenciamento de imagens, desenvolvido com base em um conjunto de ferramentas computacionais de código aberto e uso livre chamado dcm4che2. Materiais e Métodos: 0 modelo de integração é composto por um servidor de processamento de imagens e por serviços de comunicação. 0 gerenciamento de dados segue o fluxo de trabalho definido pelo perfil de pós-processamento (PWF) do Integrating the Healthcare Enterprise (IHE) e utiliza a funcionalidade de captura secundária do DICOM. Uma aplicação para lesões difusas de pulmão foi utilizada para prova de conceito. Resultados: 0 algoritmo de classificação de padrões apresentou acurácia de $78 \%$, com base em um método de teste de validação cruzada. A integração possibilita a visualização das imagens processadas como uma nova série dentro do estudo original. Conclusão: 0 modelo de integração proposto baseiase em perfis do IHE e permite o estabelecimento de procedimentos padronizados. Os princípios utilizados para integração do fluxo de trabalho são aplicáveis para qualquer tarefa não interativa de pós-processamento de imagens. Unitermos: Processamento de imagens médicas; Sistemas de arquivamento e comunicação de imagens; Captura secundária do DICOM; Perfis de integração do IHE.

Abstract Objective: This paper presents a model for integration of computer-aided diagnosis algorithms into the picture archiving and communication systems workflow that has been developed on the basis of the dcm4che 2 open source toolkit. Materials and Methods: The proposed integration model consists of an image processing server and communication services. The data management follows the workflow defined by the post-processing workflow profile (PWF) developed by Integrating the Healthcare Enterprise (IHE) and utilizes the DICOM secondary capture functionality. An application for diffuse lung disease has been utilized as proof of concept. Results: Based on a cross validation method, the standard classification algorithm presented $78 \%$ accuracy. The integration enables the visualization of processed images as a new series in the original study. Conclusion: The proposed integration model is based on IHE profiles and allows the establishment of standardized procedures. The principles used to integrate the workflow are applicable to any noninteractive post-processing task.

Keywords: Computer-aided image processing; Picture archiving and communication systems; DICOM secondary capture; IHE.

Salomão SC, Azevedo-Marques PM. Integrando ferramentas de auxílio ao diagnóstico no sistema de arquivamento e comunicação de imagens. Radiol Bras. 2011 Nov/Dez;44(6):374-380.

\section{INTRODUÇÃO}

A aplicação de sistemas de informação para gerenciamento de imagens e informações clínicas começou a ser estudada de forma mais efetiva no final da década de 80 , quando os processos de aquisição digital começaram a ser utilizados em maior

* Trabalho realizado no Centro de Ciências das Imagens e Física Médica do Hospital das Clínicas da Faculdade de Medicina de Ribeirão Preto da Universidade de São Paulo (CCIFM/ HC-FMRPUSP), através do Programa de Pós-Graduação Interunidades em Bioengenharia (EESC/IQSC/FMRP) da Universidade de São Paulo, Ribeirão Preto, SP, Brasil. Apoio financeiro: Conselho Nacional de Desenvolvimento Científico e Tecnológico (CNPq).

1. Mestre em Bioengenharia, Assessor independente em Informática Médica, Ribeirão Preto, SP, Brasil. escala nos hospitais ${ }^{(\mathbf{1})}$. Até então, cada equipamento era considerado um sistema isolado, estando conectado somente à sua estação de trabalho e a uma determinada impressora. Porém, o crescimento do uso da informação em formato digital criou a necessidade de se estabelecer uma estrutura computacional que possibilitasse a troca de

2. Livre-docente, Professor Associado do Departamento de Clínica Médica da Faculdade de Medicina de Ribeirão Preto da Universidade de São Paulo (FMRPUSP), Ribeirão Preto, SP, Brasil.

Endereço para correspondência: Dr. Paulo Mazzoncini de Azevedo Marques. Departamento de Clínica Médica, FMRPUSP. Avenida Bandeirantes, 3900, Monte Alegre. Ribeirão Preto, SP, Brasil, 14049-900. E-mail: pmarques@fmrp.usp.br

Recebido para publicação em 10/8/2011. Aceito, após revisã̃o, em 24/10/2011. dados de imagens de forma consistente e automática dentro do ambiente hospitalar. Em resposta a essa necessidade surgiu o conceito de picture archiving and communication system (PACS). O PACS é um sistema de arquivamento e comunicação voltado para o diagnóstico por imagem, que permite o pronto acesso às imagens médicas em formato digital em qualquer setor de um hospital ${ }^{(2)}$. O PACS se tornou rapidamente a opção primeira para o gerenciamento de imagens nos ambientes médicohospitalares e, em conjunto com os sistemas de informação em radiologia (radiology information system - RIS) e de informação hospitalar (hospital information 
system - HIS), forma a base para um serviço de radiologia sem filme (filmless). Um aspecto fundamental dentro do fluxo de trabalho em um ambiente radiológico digital (sem filme) é a garantia da consistência da informação que é transmitida componente a componente, dentro da cadeia de eventos presente na dinâmica de processos. Para que a consistência seja garantida, a distribuição da informação é feita segundo uma estrutura hierárquica baseada em uma distribuição top-down, ou seja, as informações são propagadas a partir do sistema de informação mais geral (HIS), passando pelo sistema de informação intermediário (RIS), até alcançar o sistema de informação mais específico (PACS). Para que isso seja possível, dois pré-requisitos são necessários: uma estrutura de rede adequada (redundante e balanceada) e padrões de comunicação bem definidos. Na radiologia digital, o padrão de comunicação principal é o DICOM (digital imaging and communications in medicine $)^{(3)}$. O DICOM é o padrão global para transferência de imagens radiológicas e outras informações médicas entre computadores. O DICOM atual, publicado em 1993 e geralmente identificado como 3.0, evoluiu das versões anteriores de um padrão desenvolvido pelo Colégio Americano de Radiologia (American College of Radiology - ACR) em conjunto com a Associação Nacional de Fabricantes de Equipamentos Elétricos dos Estados Unidos da América (National Electrical Manufacturers Association - NEMA) (ACR-NEMA 1.0, de 1985 e ACR-NEMA 2.0, de 1988). A conectividade prevista pelo padrão é muito importante no que diz respeito à razão custo/benefício para áreas da saúde que fazem uso de imagens médicas. Usuários DICOM podem prover serviços de radiologia entre instalações localizadas em diferentes regiões geográficas, aproveitar recursos de tecnologia da informação já existentes e manter os custos baixos através da compatibilidade e interoperabilidade de novos equipamentos e sistemas ${ }^{(4)}$.

Embora os avanços tecnológicos tenham possibilitado a difusão dos sistemas de gerenciamento de imagens de forma mais intensa na área médica nos últimos anos, de modo que em algumas situações o PACS seja considerado um simples objeto de consumo (commodity) ${ }^{(\mathbf{5})}$, dentro de um modelo de maturidade tecnológica ${ }^{(6)}$ ainda existem alguns aspectos que merecem uma maior investigação e desenvolvimento. Questões relacionadas aos processos de aquisição da imagem digital ${ }^{(7)}$, de distribuição, visualização $^{(8)}$ e interpretação ${ }^{(9)}$ ainda são objetos de estudo, particularmente em seus aspectos econômicos e de uso de soluções computacionais livres e de código aberto ${ }^{(\mathbf{1 0 , 1 1})}$. Destaca-se, ainda, a integração de ferramentas de auxílio ao diagnóstico dentro do fluxo de trabalho dos sistemas gerenciadores de imagens. Diagnóstico auxiliado por computador (computer-aided diagnosis CAD) pode ser definido como um diagnóstico feito por um radiologista que utiliza o resultado de análises quantitativas automatizadas de imagens radiográficas como uma segunda opinião para a tomada de decisões diagnósticas ${ }^{(\mathbf{1 2})}$. A finalidade do CAD é melhorar a precisão do diagnóstico, bem como a consistência da interpretação da imagem radiológica, mediante o uso da resposta do computador como referência ${ }^{(13-15)}$. O padrão para a utilização de sistemas CAD, ainda hoje, permanece sendo baseado no modelo de estação de trabalho isolada (standalone), sem a integração dos resultados obtidos aos sistemas de informação ou ao PACS. A integração de procedimentos de processamento de imagens dentro do fluxo de trabalho do PACS tem sido objeto recente de pesquisas na área de informática de imagens médicas ${ }^{(\mathbf{1 3}, 16-18)}$.

$\mathrm{O}$ artigo aqui apresentado descreve um modelo para integração de ferramentas de auxílio ao diagnóstico no ambiente de gerenciamento de imagens, com o objetivo de otimizar a análise visual por parte do observador humano (radiologista) dentro do fluxo de trabalho em diagnóstico por imagem. O modelo foi desenvolvido usando um conjunto de ferramentas abertas (opensource) denominado dcm 4 che2. O gerenciamento de dados segue o fluxo de trabalho definido pelo perfil de pós-processamento (PWF) do Integrating the Healthcare Enterprise (IHE) e utiliza a funcionalidade de captura secundária do DICOM (DICOM secondary capture). O dcm4che2 é uma implementação do padrão DICOM de alto desempenho, desenvolvida em linguagem de programação Java, que fornece suporte para os perfis de integração do IHE (http:// www.dcm4che.org). O IHE é uma inicia- tiva da Sociedade de Radiologia da América do Norte (Radiology Society of North America - RSNA) que tem por finalidade promover a integração dos sistemas dentro do ambiente de cuidado da saúde. O objetivo é melhorar a eficiência e a eficácia da prática clínica através da melhoria do fluxo de informação com base nos padrões de comunicação. O IHE estabelece um conjunto de arcabouços técnicos (frameworks) que define como os padrões devem ser implementados de maneira a satisfazer as necessidades da prática clínica. Utiliza três conceitos básicos: atores, transações e perfis de integração. Atores são as funcionalidades que fazem a comunicação entre os sistemas. Transações são as mensagens trocadas entre os sistemas. Perfis de integração são agrupamentos de atores e transações que compõem um fluxo específico de trabalho ${ }^{(3)}$. Como prova de conceito, foi implementada a integração de um protótipo de sistema para auxílio ao diagnóstico diferencial de lesões difusas de pulmão em exames de tomografia computadorizada junto ao sistema de gerenciamento de imagens do centro de diagnóstico por imagens de um hospital-escola.

\section{MATERIAIS E MÉTODOS}

Um ambiente computacional de testes foi construído para validar o modelo de integração entre o sistema de auxílio do diagnóstico (CAD) e o sistema gerenciador de imagens médicas (PACS). Um programa para classificação de lesões difusas de pulmão em exames de tomografia computadorizada de alta resolução, desenvolvido localmente, foi selecionado para desempenhar o papel de uma solução CAD. O sistema gerenciador de imagens médicas escolhido foi o servidor DICOM Conquest (Conquest, 2010), e para visualização dos resultados da integração CAD-PACS utilizou-se o visualizador de imagens médicas K-PACS (K-PACS, 2010). Todos os equipamentos do ambiente foram conectados em rede local (LAN) de $100 \mathrm{Mbit} / \mathrm{s}$.

A partir de uma série de pacotes de software definidos pelo modelo de integração, foi concebido um programa de computador cliente denominado Client-CAD. Este programa atua como uma entidade intermediária entre o PACS e o CAD e tem como 
objetivo efetuar a integração entre os dois sistemas através do protocolo de comunicação DICOM. O Client-CAD também agrega um componente de software conhecido pelo nome i-CAD, utilizado especialmente para alterar o número de série de imagens médicas no formato digital ${ }^{(19)}$. Ainda de forma transparente, o cliente fornece interfaces que permitem o acoplamento de módulos específicos de processamento de imagens para outras modalidades de aquisição, como mostra a Figura 1.

No ambiente de testes, o sistema CAD foi instalado e colocado em operação dentro de um computador individual com seu próprio recurso de hardware e software. Para que fosse possível estabelecer uma conexão direta entre o CAD e o PACS, o software cliente Client-CAD também foi instalado nesse mesmo computador. Uma vez ativado, o cliente interage periodicamente com o PACS através de mensagens DICOM para consulta de informação e recuperação de imagens. Por exemplo, a mensagem DICOM C-Find exprime uma consulta em um banco de dados baseada nas informações contidas no dicionário de dados do padrão, e pode ou não conter filtros para retornar resultados mais específicos. No caso do experimento aqui descrito, o cliente foi configurado para consultar apenas exames de modalidade CT (tomografia computadorizada) e região anatômica CHEST (tórax). Portanto, quando o servidor PACS recebe um novo exame de HRCT de tórax, o cliente solicita ao PACS uma cópia desse exame através do envio de mensagens de pesquisa e recuperação de imagens (query \& retrieve). As imagens recuperadas são então processadas uma de cada vez pela rotina de processamento do sistema CAD.

A rotina de processamento do CAD é composta de cinco passos: pré-processamento, segmentação, extração de características, classificação e pós-processamento. No pré-processamento os dados da imagem (pixels) são extraídos do arquivo DICOM. No passo seguinte, os pulmões são segmentados utilizando-se um algoritmo iterativo para limiarização de imagens ${ }^{(\mathbf{2 0})}$ e depois a imagem resultante é demarcada por regiões de interesse (ROIs) de $20 \times 20$ pixels. Durante o passo de classificação, cada ROI é definida como pertencente a uma das sete classes de caracterização de lesões inters-

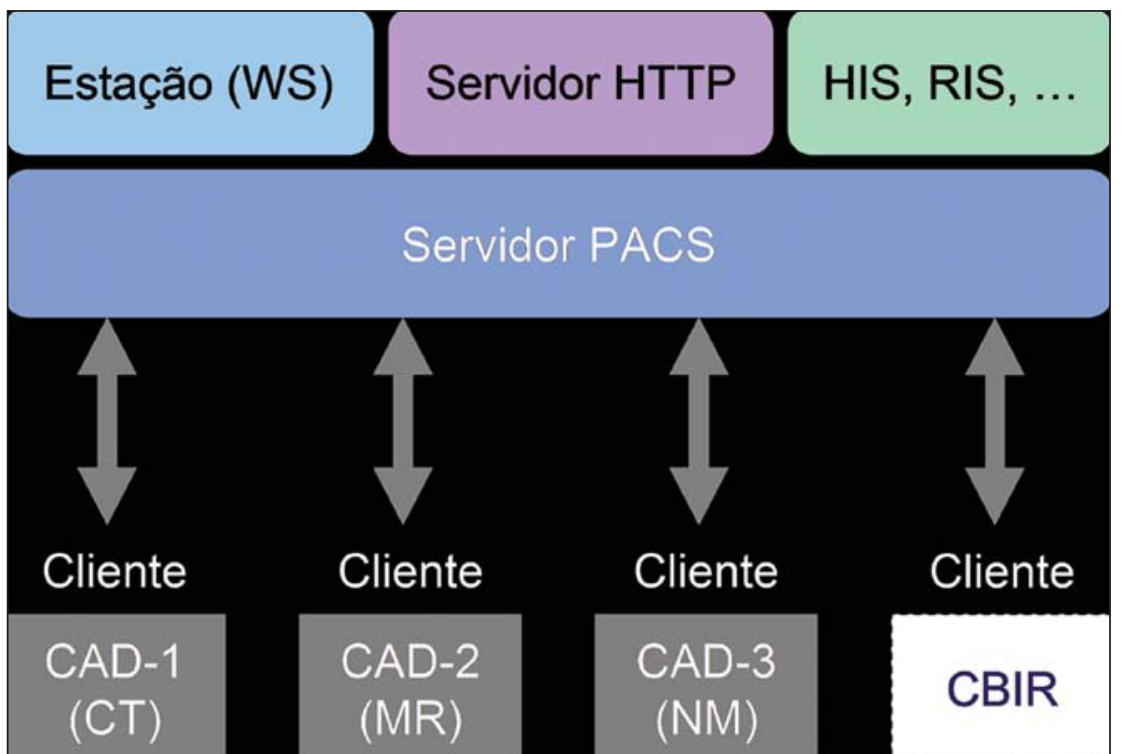

Figura 1. Arquitetura do modelo de integração. Esquema que apresenta a arquitetura do modelo de integração e seus principais componentes. As aplicações CAD se comunicam com o servidor PACS através dos serviços de comunicação fornecidos pelo cliente Client-CAD. ticiais de pulmão abordadas: normal, vidro fosco, reticular, nodular, favo de mel, enfisematoso e consolidação. No passo de pósprocessamento, cada ROI recebe uma coloração que identifica sua classe de caracterização e um novo arquivo DICOM com o resultado do processamento é gerado.
A integração CAD-PACS é concluída quando o cliente, através do componente de software i-CAD, modifica o número de série do novo arquivo DICOM e o envia de volta para o servidor PACS. A Figura 2 apresenta o princípio de funcionamento do programa Client-CAD.

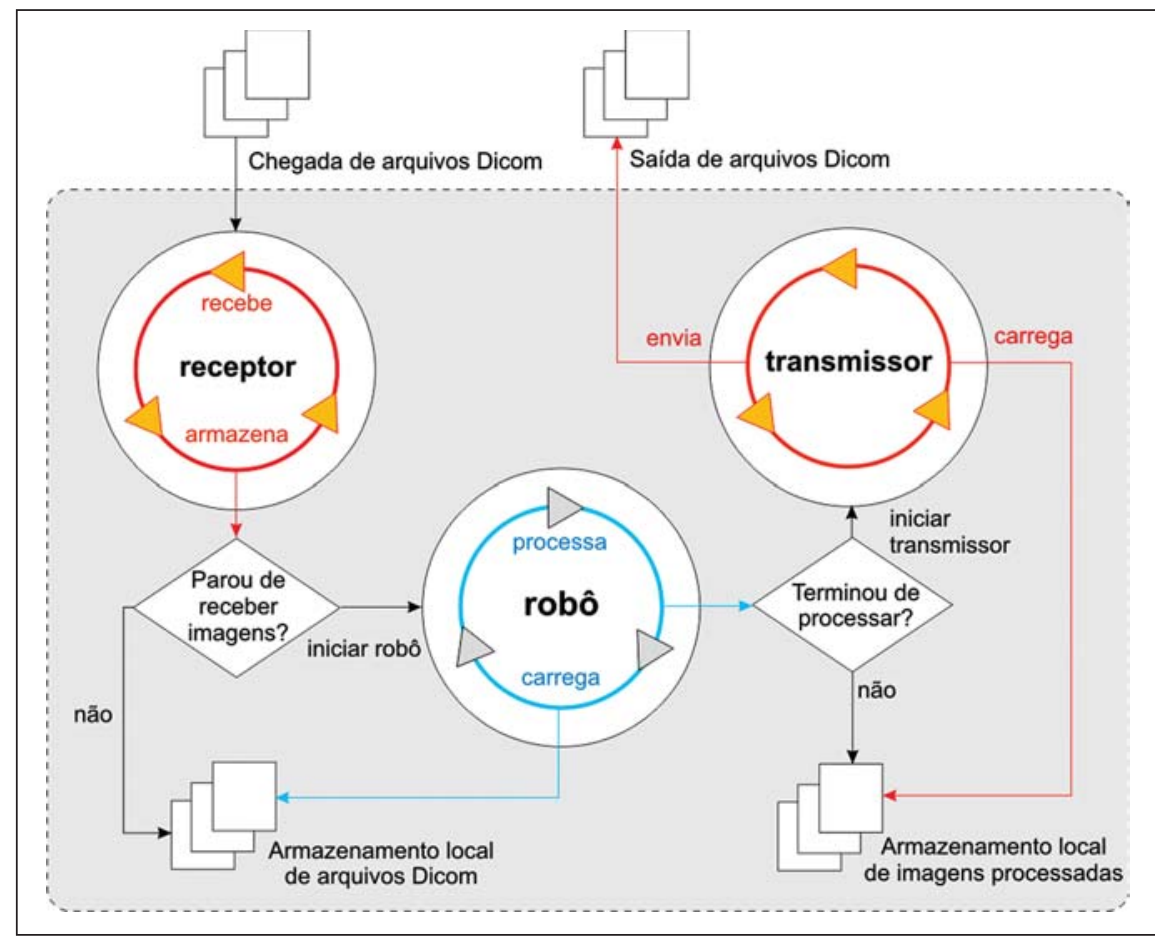

Figura 2. Esquema de funcionamento Client-CAD. Princípio de funcionamento do programa de computador Client-CAD concebido a partir do modelo integração CAD-PACS. Ilustra o funcionamento dos processos receptor, robô e transmissor responsáveis pelo processamento e envio dos resultados CAD para o PACS. 
Com base na arquitetura de funcionamento do cliente, o primeiro processo chamado receptor representa um programa de computador independente que implementa um provedor de serviço DICOM para armazenamento de imagens (storage service class provider-CStore SCP). Ele entra em ação sempre que o cliente solicita uma cópia de um determinado exame armazenado no PACS. Para cada imagem recebida, o cliente invoca o processo robô, que por sua vez inicia a rotina de processamento de imagens do sistema CAD. Quando a rotina CAD é finalizada, o robô armazena localmente a imagem resultante do processamento e inicia o terceiro e último processo, o transmissor. Nessa etapa, o i-CAD é utilizado para alterar o número de série da imagem processada. Finalizando o ciclo de integração, o transmissor envia mensagens DICOM para solicitação de armazenamento (storage service class user - CStore $\mathrm{SCU})$ de imagens e transmite a imagem resultante para o servidor PACS.

\section{RESULTADOS}

Os resultados do experimento estão diretamente relacionados com o desenvolvimento do software Client-CAD e ao su- cesso da integração do sistema para auxílio ao diagnóstico de lesões difusas de pulmão junto ao sistema de gerenciamento de imagens. O fluxo de trabalho do programa Client-CAD contempla quatro tarefas vitais que contribuíram para o êxito da integração:

- pesquisa e recuperação: o cliente consulta o servidor PACS periodicamente, pesquisando por novos exames realizados através do envio de mensagens DICOM CFind. A consulta sempre é realizada utilizando-se o modelo de informação para pesquisa e recuperação de imagens em nível de estudo (study root query retrieve information model FIND);

- processamento CAD: uma vez recebidas e armazenadas, as imagens são processadas uma de cada vez pela rotina CAD iniciada pelo cliente. Para cada imagem, o resultado do processamento consiste de uma imagem JPEG que contém informações relevantes que podem auxiliar na tomada de decisão do médico radiologista;

- encapsulamento: cada imagem JPEG resultante é encapsulada pelo cliente em um formato DICOM compatível. Informações obrigatórias do dicionário, como, por exemplo, identificação e nome do paciente, são copiadas do arquivo DICOM original.
A diferença entre o arquivo original e a versão encapsulada está na forma de construção do arquivo. $\mathrm{O}$ arquivo DICOM encapsulado é codificado a partir de uma imagem JPEG e segue o modelo de informação da classe de captura secundária (DICOM secondary capture image storage SOP class);

- transmissão: a transmissão das imagens-resultado é realizada através do envio da mensagem DICOM C-Store SCU implementada no cliente. Dessa forma, quando o servidor PACS recebe o arquivo DICOM encapsulado, o gerenciador de arquivamento armazena seu conteúdo na mesma pasta do paciente do arquivo original, mas dentro de uma nova série de imagens de acordo com o novo número de série gerado pelo programa i-CAD.

A Figura 3 apresenta a interface do programa Client-CAD. É possível visualizar vários dos parâmetros utilizados. Os campos no canto superior esquerdo são usados para configurar a comunicação DICOM entre o cliente e o servidor PACS. Logo abaixo estão os campos que identificam o PACS que será consultado. Ainda abaixo, o último bloco estabelece as configurações de agendamento de consultas do cliente para pesquisa e recuperação de imagens.

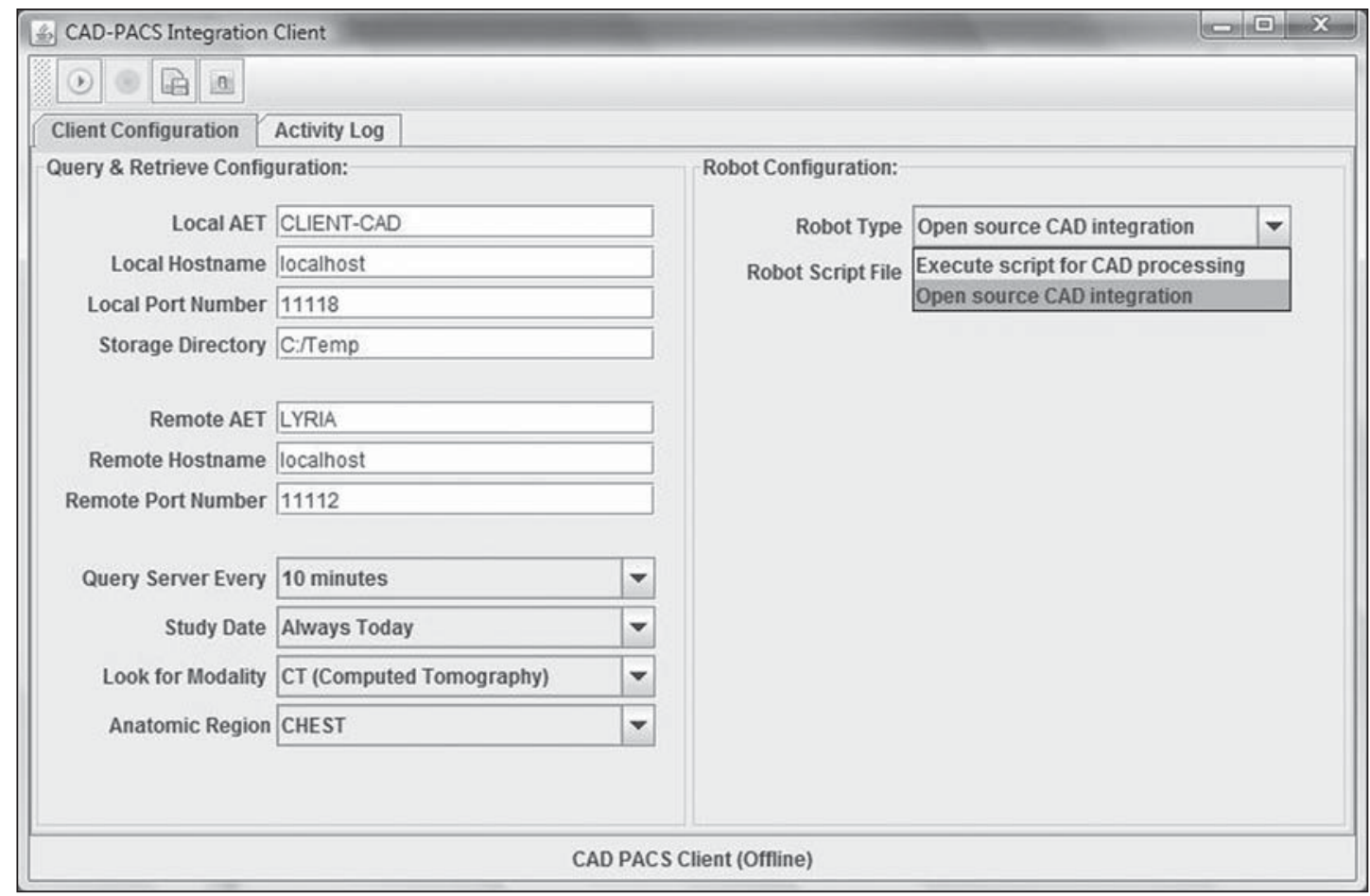

Figura 3. Interface Client-CAD. Programa Client-CAD. Interface para configurar a comunicação com o servidor PACS. 
Para ilustrar a comunicação CAD-PACS, a Figura 4 mostra a interação entre o cliente e o servidor PACS Conquest. No destaque é possível visualizar uma entidade de nome "CLIENT-CAD" solicitando o armazena- mento de uma imagem, resultado do processamento CAD. Uma vez estando as imagens-resultado armazenadas corretamente no servidor PACS, é possível visualizar a nova série criada pela integração
CAD-PACS na estação de visualização. As Figuras 5 e 6 mostram o visualizador KPACS apresentando exemplos de séries de imagens originais ao lado da correspondente série processada pelo sistema CAD.

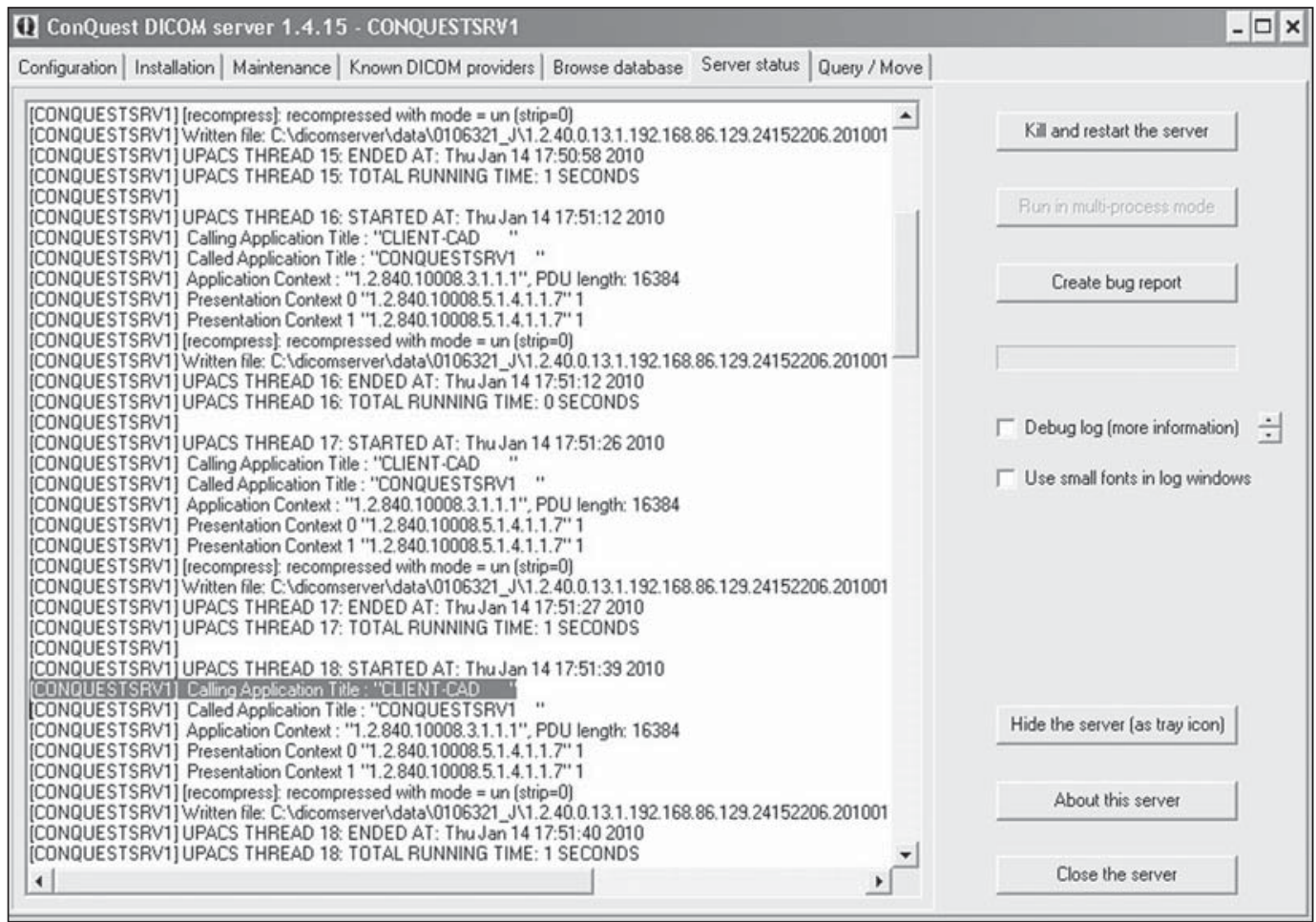

Figura 4. Interface servidor DICOM. Interface do servidor Conquest para visualização de eventos e status. No destaque é possível visualizar a interação do servidor com o programa Client-CAD.

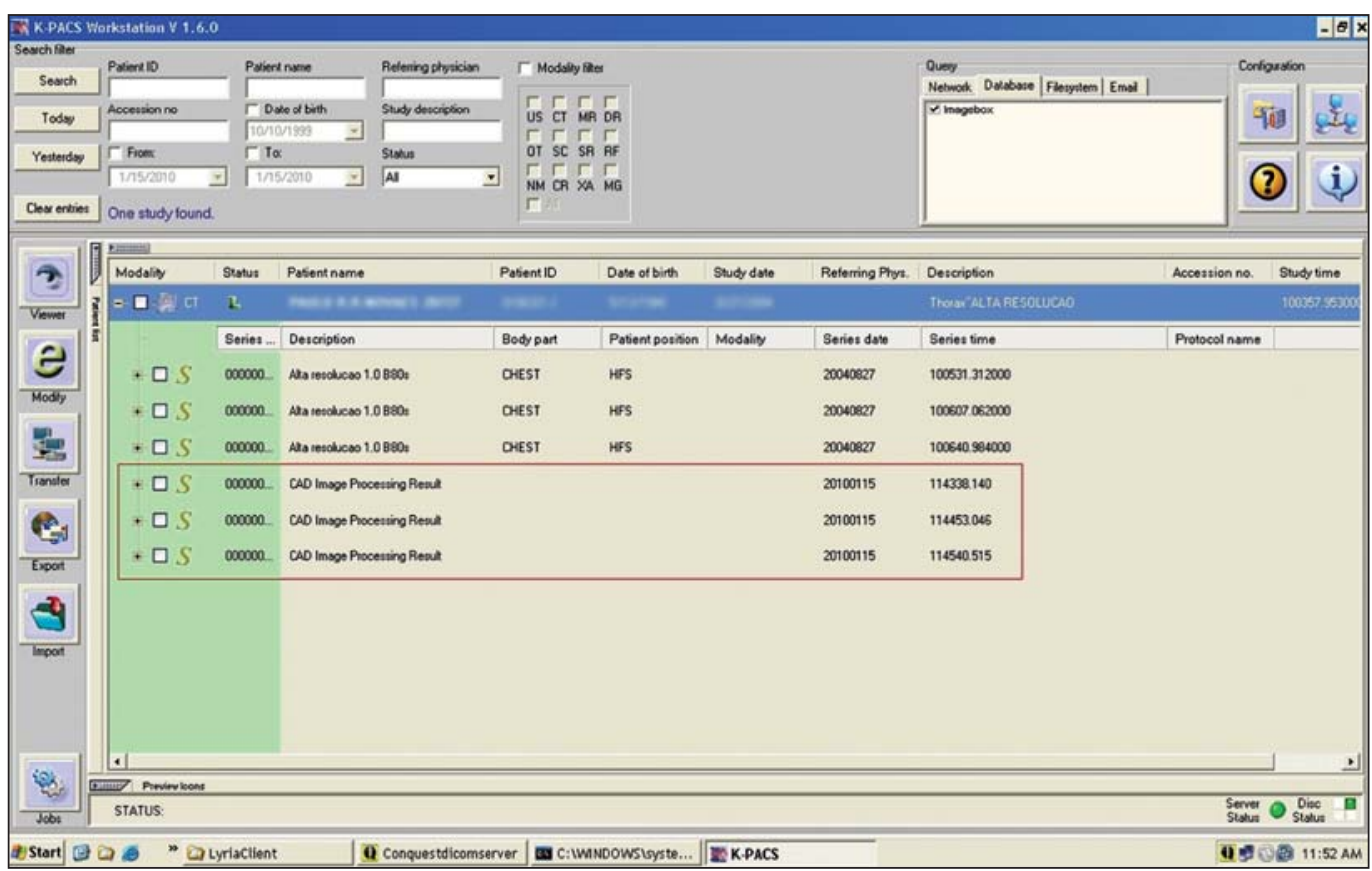

Figura 5. Lista de séries de estudo atualizada. Interface de pesquisa do visualizador K-PACS. No destaque, três novas séries de imagens processadas depois de concluído o processo de integração CAD-PACS. 


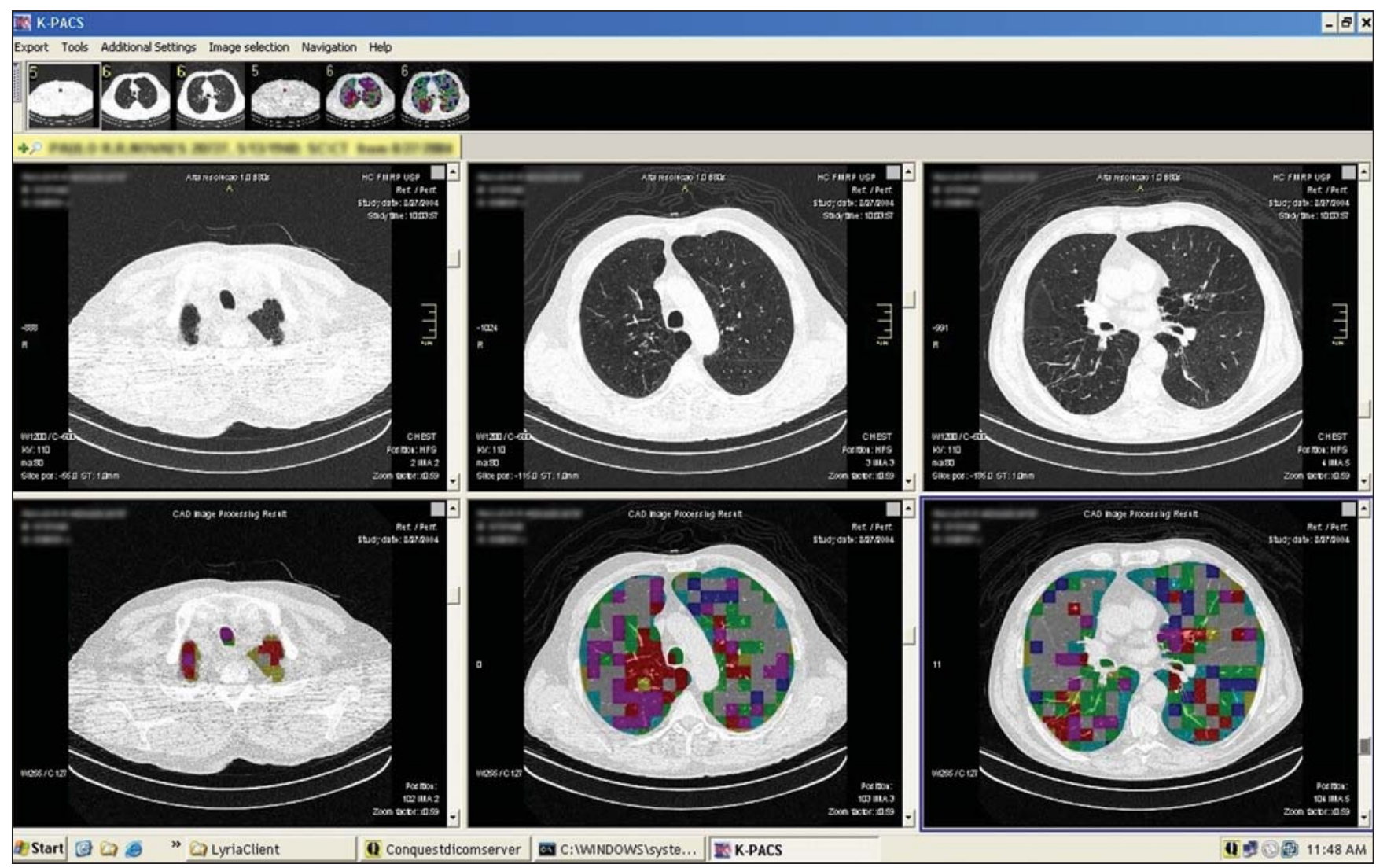

Figura 6. Exemplo de tela de visualização. Módulo de visualização do K-PACS após a integração CAD-PACS. A porção superior apresenta as séries de imagens originais do paciente. Abaixo, as séries correspondentes com as imagens processadas pelo CAD.

\section{DISCUSSÃO}

Os testes iniciais mostraram bom potencial do modelo proposto para a integração CAD-PACS, porém uma limitação importante diz respeito ao tipo dos arquivos utilizados durante o fluxo de trabalho da integração. Os formatos atualmente suportados são o DICOM e o JPEG. Portanto, o modelo de integração é compatível com aplicações CAD que forneçam uma imagem JPEG como resultado do processamento. Deve-se destacar que a imagem JPEG encapsulada no DICOM não apresenta a mesma qualidade diagnóstica da imagem original, devido a perdas inerentes ao próprio formato, devendo ser utilizada como uma informação complementar na tomada de decisão feita a partir da inspeção visual da série original. Caso o CAD não forneça saída em formato JPEG, uma solução seria capturar e encapsular a imagem da tela do computador no momento em que o resultado é apresentado. Essa alternativa simples, no entanto, pode de certa forma comprometer a qualidade da leitura dos resultados e atrapalhar a interpretação dos dados por parte do médico, devido aos vários elementos visuais e textuais presentes na tela do computador que formam a imagem capturada. De modo geral, sistemas CAD nem sempre apresentam seus resultados apenas na forma de imagem. É comum encontrar dados quantitativos distribuídos em planilhas, gráficos, documentos HTML e até relatórios completos no formato de arquivo PDF. São dados relevantes que, se integrados ao exame do paciente no servidor PACS, podem fornecer um conjunto valioso de informações para auxiliar na tomada de decisão. Tal integração pode ser realizada utilizando-se métodos disponibilizados pelo DICOM structured reporting (SR), uma nova extensão do padrão DICOM que estabelece padrões para a codificação de documentos que incorporam referências para imagens médicas e dados relacionados, bem como serviços para a transmissão e troca de informação. Um modelo de integração baseado nas propriedades do DICOM-SR seria mais completo e possibilitaria inclusive atividades interativas de recuperação de informação, como, por exemplo, recuperação de imagens por conteúdo (contente-based image retrieval - $\mathrm{CBIR})$.

A contribuição real de uma ferramenta de auxílio ao diagnóstico no processo de tomada de decisão depende de uma sinergia entre o profissional e a ferramenta. Essa interação sinérgica não ocorre, em geral, de forma natural, demandando um trabalho de treinamento do usuário e de adequação da solução computacional. O trabalho aqui descrito apresenta um modelo de integração de ferramentas de processamento de imagens de forma transparente dentro do fluxo de trabalho de um ambiente radiológico digital. Porém, a avaliação da contribuição efetiva da solução implementada como ferramenta de apoio à tomada de decisão, com base em testes de observador para medida da acurácia diagnóstica, ficou fora do escopo da investigação aqui apresentada. 


\section{CONCLUSÃO}

Com a disseminação massiva de equipamentos digitais para geração de imagens médicas, em conjunto com a adoção de soluções de gerenciamento de informação (PACS), torna-se cada vez mais comum a existência de serviços de diagnóstico por imagem que funcionam sob um modelo de radiologia sem filme. No ambiente sem filme, as imagens são armazenadas e distribuídas com base nas funcionalidades do padrão DICOM. O processamento de imagens visando o auxílio computadorizado ao diagnóstico tem-se mostrado como uma importante área de pesquisa nos últimos anos, inclusive com o surgimento de soluções comerciais aprovadas para uso clínico pela Food and Drug Administration (FDA). Nesse cenário, a possibilidade de integração das ferramentas de auxílio ao diagnóstico no ambiente de gerenciamento de imagens médicas pode contribuir para otimizar a atividade do médico especialista, possibilitando o acesso aos dados computacionais de forma integrada e transparente. $\mathrm{O}$ trabalho aqui apresentado descreve um modelo de integração desenvolvido com base em bibliotecas DICOM de uso livre. Embora focado no auxílio ao diagnóstico diferencial de lesões pulmonares difusas em exames de tomografia computadorizada, o arcabouço implementado pode ser facilmente estendido a outras aplicações computacionais automáticas que não necessitem de interação com o usuário. A extensão para outras aplicações dependeria, es- sencialmente, da adequação da rotina de processamento de imagens para a nova área, segundo a complexidade e variação de seus padrões.

\section{REFERÊNCIAS}

1. Wiley G. The prophet motive: how PACS was developed and sold. Imaging Economics - May 2005. [acessado em 8 de agosto de 2011. Disponível em: http://www.imagingeconomics.com/ issues/articles/2005-05_01.asp

2. Siegel EL, Kolodner RM. Filmless radiology: state of the art and future trends. In: Siegel EL, Kolodner RM, editors. Filmless radiology. New York, NY: Springer-Verlag; 1999. p. 3-20.

3. Azevedo-Marques PM, Salomão SC. PACS: sistemas de arquivamento e distribuição de imagens. Rev Bras Fís Méd. 2009;3:131-9.

4. Horii SC. A nontechnical introduction to DICOM. RSNA Informatics. [acessado em 8 de agosto de 2011]. Disponível em: http://www.rsna. org/technology/dicom/intro/index.cfm

5. Chen J, Bradshaw J, Nagy P. Has the picture archiving and communication system (PACS) become a commodity? Journal of Digital Imaging. 2011;24:6-10.

6. van de Wetering R, Batenburg R. A PACS maturity model: a systematic meta-analytic review on maturation and evolvability of PACS in the hospital enterprise. Int J Med Inform. 2009;78:12740.

7. Vela JG, Bhaya A, Monteiro AMV, et al. Digitalização de filmes radiográficos com costura de imagens. Radiol Bras. 2011;44:233-7.

8. Parizoti A, Netto TG. Estudo de otimização de imagens em fluoroscopia intervencionista. Radiol Bras. 2009;42:375-8

9. Ferreira DM, Cohrs FM, Lederman HM, et al. Comparação dos tempos de geração e digitação de laudos radiológicos entre um sistema eletrônico baseado em voz sobre IP (VoIP) e um sistema tradicional baseado em papel. Radiol Bras. 2010;43:7-12.

10. Nobre LF, von Wangenheim A. Software gratuito: uma opção para o radiologista? Radiol Bras. 2010;43(5):ix-x.
11. Barra FR, Barra RR, Barra Sobrinho A. Visualizadores de imagens médicas gratuitos: é possível trabalhar apenas com eles? Radiol Bras. 2010; 43:313-8.

12. Azevedo-Marques PM. Diagnóstico auxiliado por computador na radiologia. Radiol Bras. 2001;34: 285-93

13. Doi K. Computer-aided diagnosis in medical imaging: historical review, current status and future potential. Comput Med Imaging Graph. 2007;31: 198-211.

14. Azevedo CM, Alvarenga AV, Pereira WCA, et al. Análise computacional da textura de tumores de mama em imagens por ultrassom de pacientes submetidas a cirurgia conservadora. Radiol Bras. 2009;42:363-9.

15. Pádua RDS, Oliveira LF, Azevedo-Marques PM, et al. Auxílio à detecção de anormalidade perfusional miocárdica utilizando atlas de SPECT e registro de imagens: resultados preliminares. Radiol Bras. 2008;41:397-402.

16. Caritá EC, Seraphim E, Honda MO, et al. Implementação e avaliação de um sistema de gerenciamento de imagens médicas com suporte à recuperação baseada em conteúdo. Radiol Bras. 2008; 41:331-6.

17. Azevedo-Marques PM, Ponciano-Silva M, Salomão SC, et al. CAD-PACS integration: a framework for pattern recognition of diffuse lung disease in HRCT. Int J Comput Assist Radiol Surg. 2009;4 Suppl 1:S177-83.

18. Le AH, Liu B, Huang HK. Integration of computer-aided diagnosis/detection (CAD) results in a PACS environment using CAD-PACS toolkit and DICOM SR. Int J Comput Assist Radiol Surg. 2009;4:317-29.

19. Zhou Z, Liu BJ, Le AH. CAD-PACS integration tool kit based on DICOM secondary capture structured report and IHE workflow profiles. Comput Med Imaging Graph. 2007;31:346-52.

20. Bugatti PH, Ponciano-Silva M, Traina AJM, et al. Content-based retrieval of medical images: from context to perception. Computer-Based Medical Systems, 2009. CBMS 2009. 22nd IEEE International Symposium on; 2009 Aug 2-5; Albuquerque, NM. Proc CBMS. 2009; p. 1-8. 